\title{
ASSOCIATION OF CHROMOBLASTOMYCOSIS AND SUBACUTE CUTANEOUS LUPUS: CASE REPORT
}

Natália Nunes ${ }^{1}$, Edith M. M. Batista1, Renata R. Gomes², Vania Aparecida Vicente², Daniel Wagner C.L.Santos ${ }^{3}$, Matheus Cardoso Silva ${ }^{4}$, Sirlei Garcia Marques ${ }^{1,5}$, Pablo Gustavo Ribeiro Furtado ${ }^{5}$ e Conceição de M. P. S de Azevedo'1 1-UFMA, 2-UFPR, 3-UNIFESP-4-CEUMA, 5-LAB CEDRO

\section{OBJECTIVE}

To report the case of a patient with chromoblastomycosis (CBM) and subacute cutaneous lupus erythematosus (SCLE) and to evaluate the systemic inflammatory response

\section{METHODS}

This is a descriptive study of a clinical case of chromoblastomycosis in a patient, with a biopsy and blood collection. From the biopsied skin sample, culture, direct examination, histopathology and genetic sequencing of the fungus were performed to confirm the CBM. IL-6, IL-10, IFN- $\gamma$, TNF- $\alpha$ and IL-17A cytokines were quantified by Cytometric Bead Array (CBA) Mouse Th1 / Th2 / Th17 Cytokine Kit.

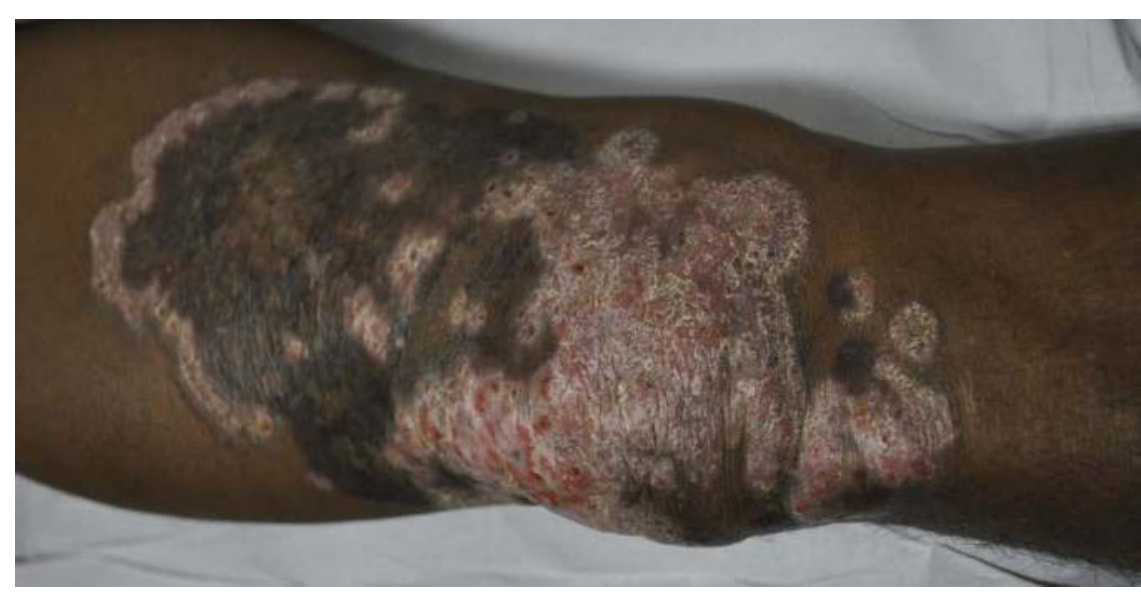

Fig 1- Cicatricial lesion with circinate borders on knee, before treatment.

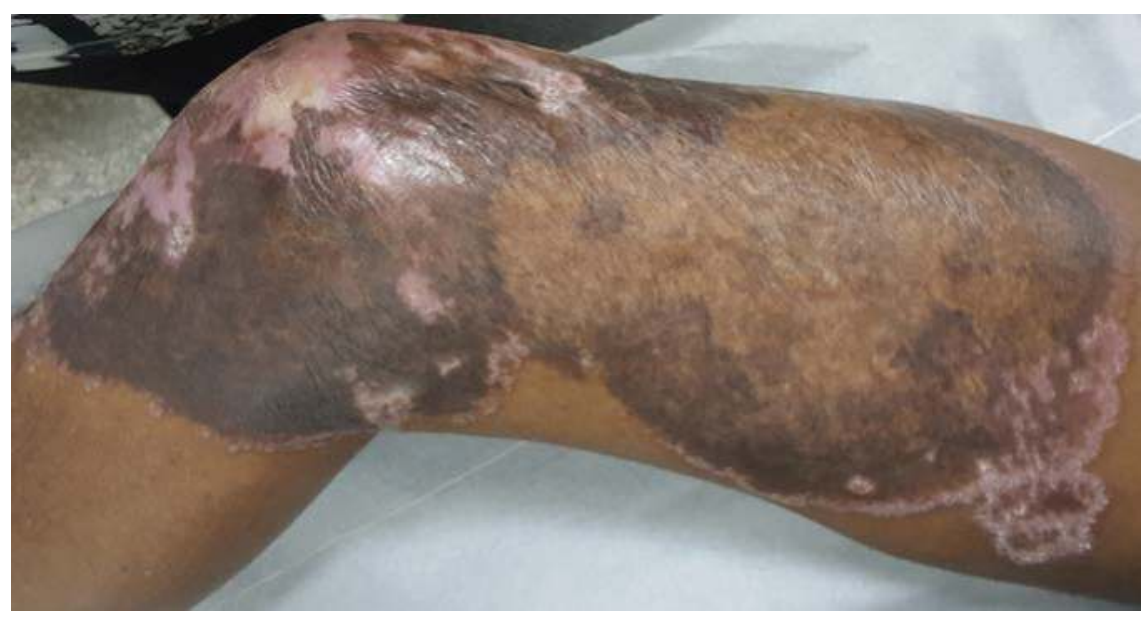

Fig 2- Cicatricial lesion with fibrosis on knee. After introduction of imiquimod.

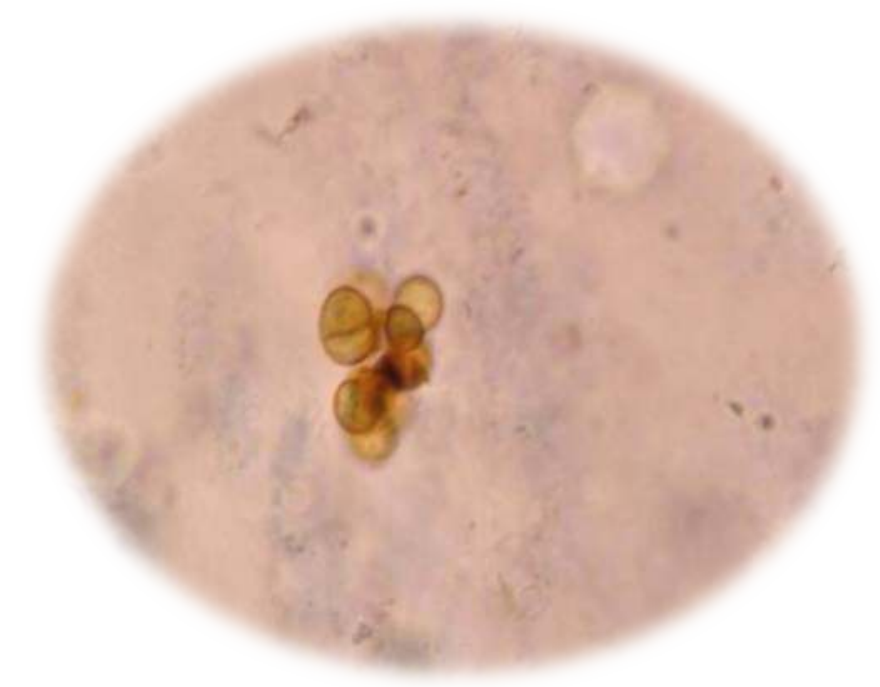

Fig 3- Muriformes cells

\section{RESULTS}

Male, 52 years old, with diagnosis of SCLE under treatment for 5 years, in the use of chloroquine and methotrexate, with disease under control. The patient presents cicatricial lesion, with circinate borders, that extended with infiltrative aspect in area of the knee, characterized also by areas of fibrosis and intense desquamation (Fig1), impeding the ambulation and the correct extension of the right lower limb. Histopathological examination of skin biopsy showed muriform cells(Fig2) with intense fibrosis and granulomas. Culture of skin biopsy was positive for black mould suggestive of $F$. pedrosoi. Molecular identification by genetic sequencing of ITS region confirmed Fonsecaea pedrosoi as causative agent(Fig3). In the evaluation of serum inflammatory response, only IL-6 was detected, which was more evident after 6 months of methotrexate suspension, from $3.47 \mathrm{pg} / \mathrm{mL}$ to 14.9 $\mathrm{pg} / \mathrm{mL}$. The initially slow therapeutic response, despite being prescribed $400 \mathrm{mg} /$ day of itraconazole, only shows reduction of the lesion after the introduction of Imiquimod (topical imumodulator)(Fig4).

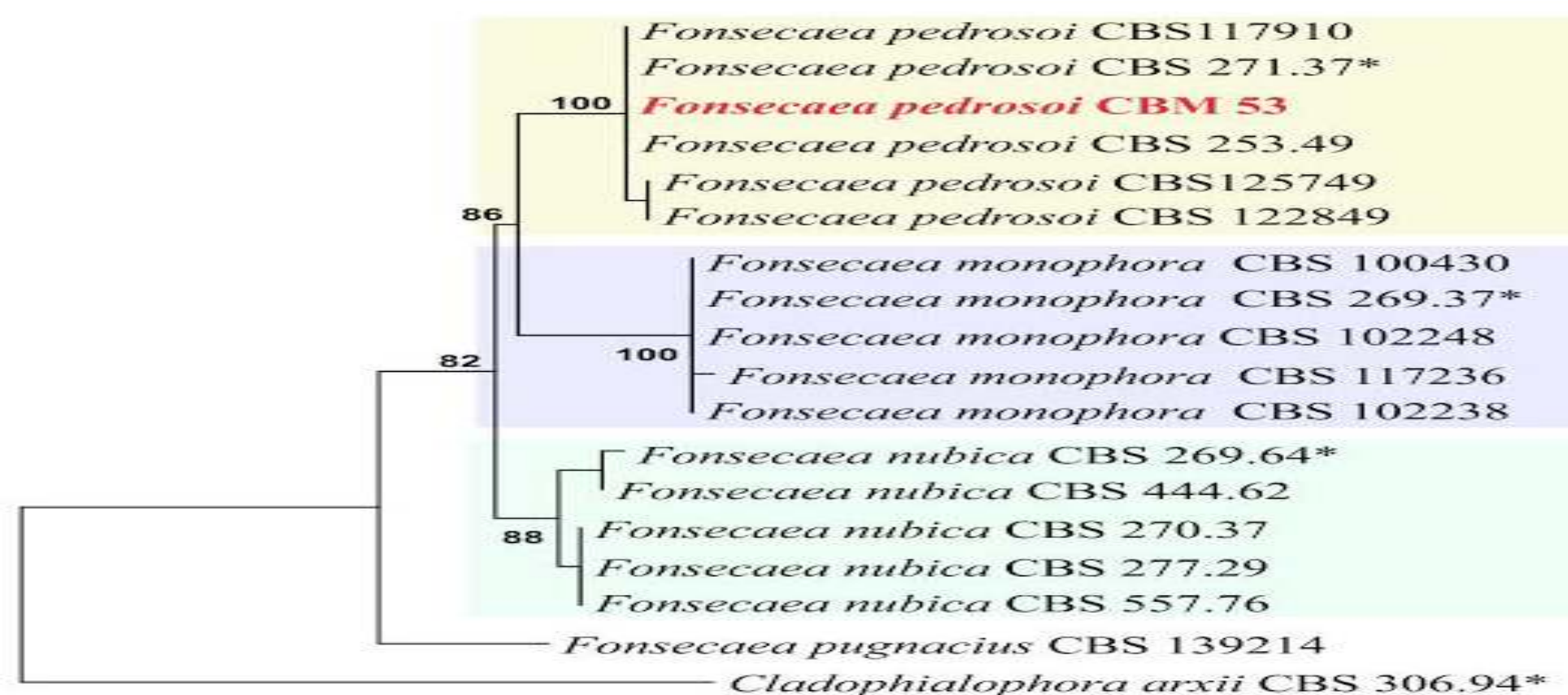

Fig 4-Phylogenetic trees of Fonsecaea and Cladophialophora based on confidently aligned ITS and partial $B T 2$ and $C D C 42$ sequences constructed with maximum likelihood implemented in MEGA 6.0.

\section{CONCLUSION}

It is the first study known to report the association between CBM and SCLE. The CBM of the patient has low therapeutic response, as expected for the severe disease, presenting better response after the introduction of Imiquimod, showing a drug with good perspective of adjuvant therapy. The causative agent found is Fonsecaea pedrosoi, the most isolated agent in Brazil. 